\title{
Environmental impact assessment from marble exploitation on surface and underground water in SAKALALINA- MADAGASCAR
}

\author{
NICOLAS Obin*, Xiuzhi Shi \\ School of Resources and Safety Engineering, Central South University, Changsha 410083, China \\ "nicolas_obin@yahoo.fr,tel: +8618711102069
}

Keywords: Marble exploitation, Environment impacts, Physico-chemical analysis, Surface and underground water, Pollution

Abstract: The determination of the impacts of marble exploitation on the surface and underground water at SAKALALINA-MADAGASCAR was determined by assessing the physico-chemical properties of the water samples obtained from SAKALALINA and its environs. The concentration of Fe, $\mathrm{Mn}, \mathrm{Cu}, \mathrm{Zn}, \mathrm{Pb}, \mathrm{Cd}, \mathrm{Hg}$, As and selected major ions in water samples were analyzed in the laboratory to assess their role in the contamination of both surface and ground water. The water in this Study area is mainly used for drinking and industrial purposes followed by agriculture to some extent.

The specific objectives are: to describe contamination process in surface water and groundwater due to mining activities; to identify and characterize the critical aspects relating to the water-related impacts of mining in the study area; to propose the best method and the risk management measures for the impacts.

The result of water quality parameters were compared with the standards laid down by United State Environmental Protection Agencies (USEPA) standard (2015) and World Health Organization (WHO), suitable for drinking and domestic purpose. The results reveal that levels for certain water quality parameters like EC, $\mathrm{pH}$, alkalinity, nitrate, phosphate exceeded the desirable limits prescribed by the USEOA and WHO standards.

The results of physico-chemical analysis of groundwater level in the study area show high pollution level in the region.

\section{Introduction}

Mining activities accelerate the rate and degree of changes in the natural environment. These activities modify landscapes and can have long-term pollution impacts on communities and water resources due to their physical degrading nature, as well as their use of chemicals and other harmful substances [6]. 
The mining product is a non-renewable resource that can be exploited on contact, or near the biosphere, which brings renewable resources and includes biodiversity. Therefore, it is necessary to reconcile the sustainable management of the environment with that of the resources of the subsoil [3].

Biodiversity in Madagascar, which has a heritage of first importance, is already under threat and it is not necessary that the mining development come to aggravate the situation [17]. Currently, mining activities are prohibited within protected areas, but the discovery of a new deposit of gems in the vicinity of these areas may endanger it. This justifies the implementation of the institutional legislative measures necessary to make all mining activities compatible with the environment.

A question arises "under which condition, such mining brings a social and economic development with preservation of the environment of such a region? ". Such a question prompts us to choose the topic entitled "ENVIRONMENTAL IMPACT ASSESSMENT FROM MARBLE EXPLOITATION ON SURFACE AND UNDERGROUND WATER IN SAKALALINAMADAGASCAR".

This work was produced as part of the study environmental impact of marble mining on water in the SAKALALINA-MADAGASCAR area. This zone is located in the mining perimeters of the company MINE TANY HAFA (MTH), it belongs to the Rural Municipality of Sakalalina, Ihosy District, Ihorombe Region. The company MTH exploits the marble.

To better define the subject, the work consists of three parts:

$\checkmark$ The first part presents the introduction;

$\checkmark$ The second part deals with the materials and methods;

$\checkmark$ The third part concerns the results and discussion;

\section{Materials and methods}

\subsection{Geographical and administrative location of study area}

The general framework of the study site is in the Ihorombe Region, from the Province of Fianarantsoa to the South Center of the Island of Madagascar. The Region is served by the National Route number seven (RN7). The Ihorombe Region is made up of three Districts: Ihosy, Iakora and Ivohibe. Our study was conducted in the Iangaty Fokotany of Sakalalina Rural District, about $56 \mathrm{~km}$ east of the city of Ihosy. 


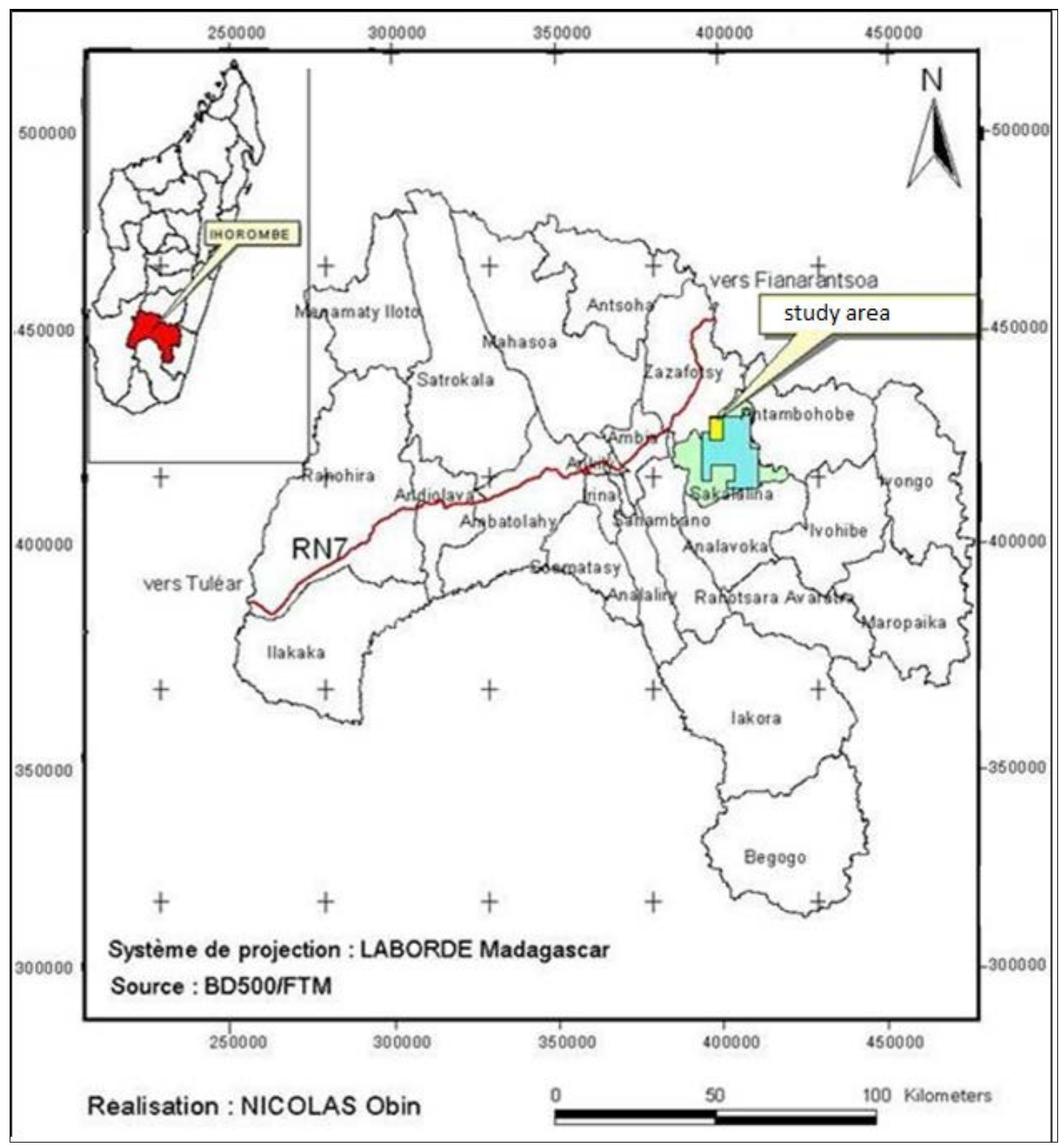

Figure 1: Location map of the study area

\subsection{Methodology and approach}

This section gives a brief description of the methods and approach that were used during this study. The strategy focuses on water quality and water pollution as well as source degradation of surface water and groundwater, among others.

Our method consists in identifying and measuring the positive or negative impacts depending on the receiving environment and the operations to be performed. So the work plan looks like this: the location of the perimeters; the description of the receiving medium; collect relevant information for the work in some institutions in Madagascar: Regional Water Administration, Ministry for Mineral Resources and Mining Company (MTH); Determination of parameters physicochemical in Water; the 
description and characterization of the operations to be performed; the identification of the impacts to be incurred; mitigation measures and the recommendations.

The Groundwater quality at different 20 well locations was selected as per their distribution along the mining belt of the study area.

The data used for the basic configuration to carry out our study are: Previous map data: Geological map, Topographic map; Bibliographical data: Literature research: different articles and books were consulted in the research questions; the result of physico-chemical analyzes; data collected in the field; satellite image (Landsat7 ETM +).

The Landsat7 ETM + image has an area of $170 \mathrm{~km} \times 185.2 \mathrm{~km}$, it has eight (8) channels whose resolution and wavelength as well as the respective characteristics are illustrated by the following table. Landsat TM channels reflect the state of the land surface, which is influenced by lithology, hydrography, grain size, soil condition, and so on. Therefore, the RGB composition is used, combining in order the channels 7, 4, 2 which give a better basic picture for the description of the study area.

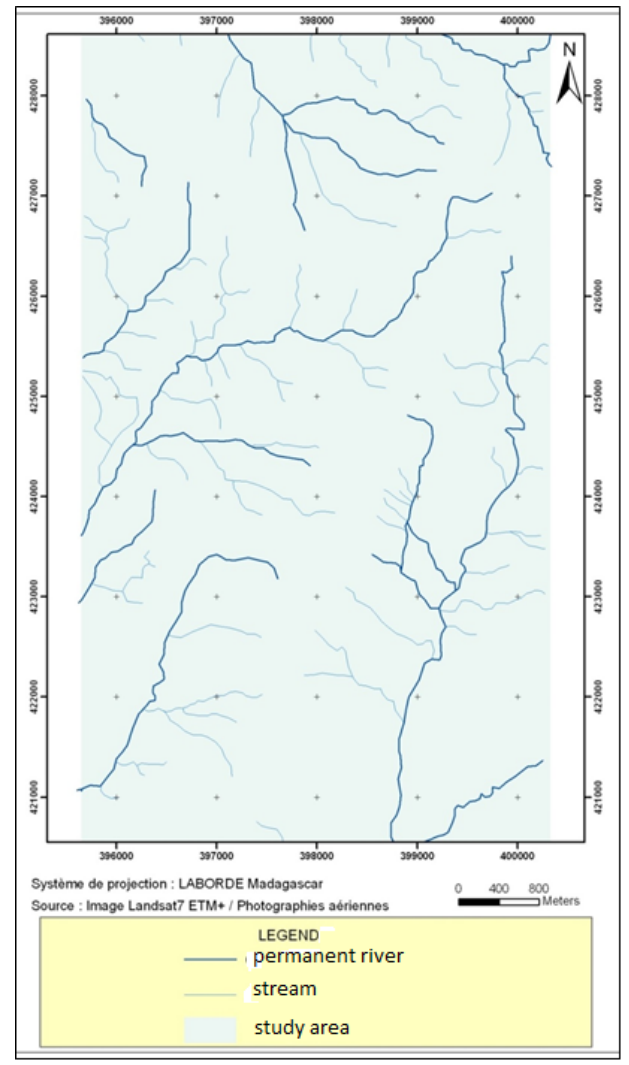

Figure 2: Map of river system 


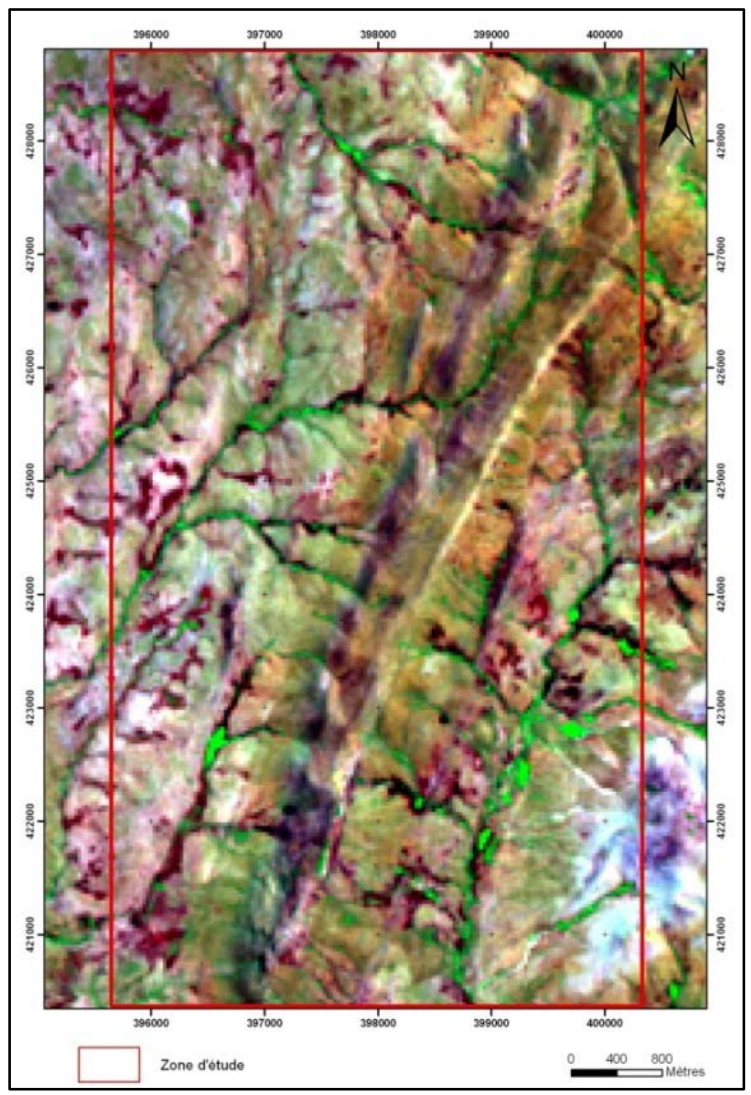

Figure3: Landsat7 ETM image +

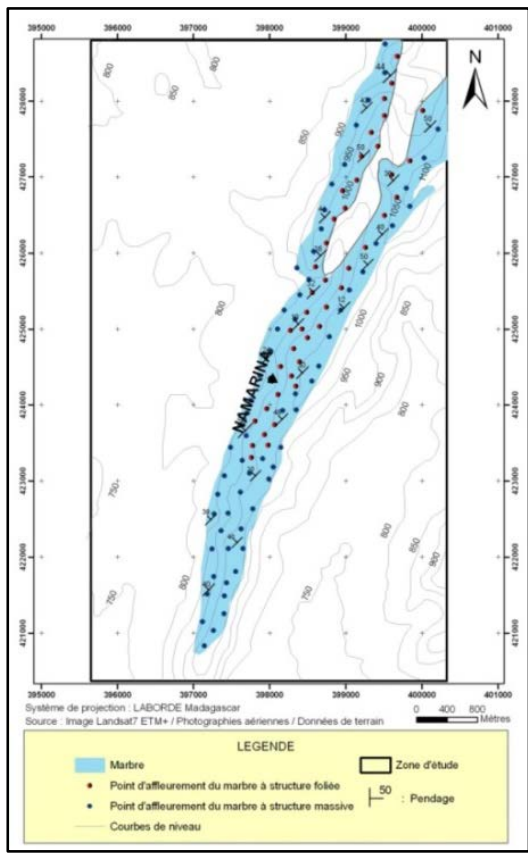

Figure 4: Map showing the outcrop points of water collected in the study area 


\subsection{Organization of the methodology}

The sequence of the different steps is illustrated in this figure.

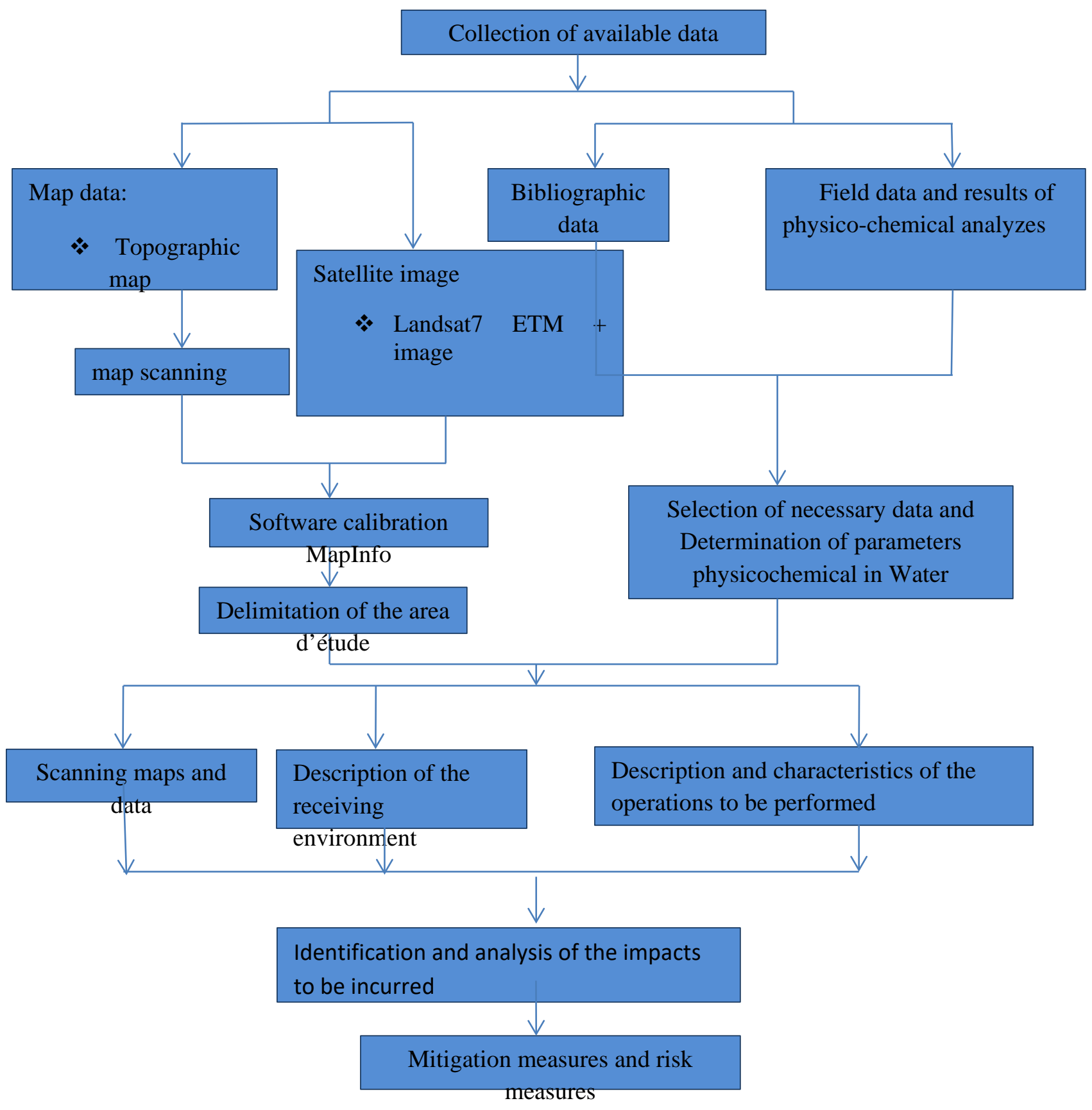

Figure 5: Organization of the methodology 


\section{Results and discussion}

\subsection{Geology of study area}

Madagascar is the product of several episodes of fragmentation and then reconstruction of Gondwana. It is geologically composed of two main entities [19]:

- Precambrian crystalline basement polystructured by successive orogenic and tectonometamorphic events. It is outcropping on the eastern two-thirds of the island;

- The sedimentary cover that rests unconformably on the crystalline base. It is from the Phanerozoic period and is known as the Phanerozoic cover of Madagascar.

The study area belongs exclusively to the crystalline basement of the southern center of Madagascar. The region has been subjected to a deep metamorphism belonging to the catzone [20]. This level of metamorphism is characterized by the following minerals: biotite, amphibole, pyroxene, and cordierite. Concerning regional tectonics, we found the succession of a synclinal structure and an anticlinal structure [16]. Our study area is framed in the tectono-metamorphic domain of Ikalamavony.

According to geological map and field observations, the geological formations in the study area consist of marble (cipolin), quartzite, gneiss, leucogneiss, pyroxenite, granitoid migmatite.

\subsection{Determination of parameters physicochemical in Sakalalina Water}

Twenty water samples (river samples, quarry pits water samples, borehole water sample) were randomly collected and analyzed. The table below represents the water quality in different points of Sakalalina water. The physicochemical, heavy metal and bacteriological analysis of the water sample, as well as the variables were compared with those of the World Health Organization (WHO) standard (2008), United State Environmental Protection Agencies (USEPA) standard (2010). 
Table 1: parameters physicochemical from Laboratory data for samples analyzed

Source : MTH Company (2016)

\begin{tabular}{|c|c|c|c|c|c|c|c|c|c|c|c|c|c|c|c|c|c|c|c|c|c|}
\hline $\begin{array}{c}\text { Sa } \\
\mathrm{m} \\
\mathrm{pl} \\
\mathrm{e} \\
\text { co } \\
\text { de }\end{array}$ & $\begin{array}{l}\mathrm{P} \\
\mathrm{h}\end{array}$ & $\begin{array}{l}\mathrm{T} \\
\mathrm{e} \\
\mathrm{m} \\
\mathrm{p} \\
\left(^{0}\right. \\
\mathrm{C} \\
)\end{array}$ & $\begin{array}{l}\mathrm{T} \\
\mathrm{D} \\
\mathrm{S} \\
( \\
\mathrm{m} \\
\mathrm{g} / \\
\mathrm{l} /\end{array}$ & $\begin{array}{c}\text { Co } \\
\text { nd } \\
(\mu \\
S / \\
\text { cm } \\
)\end{array}$ & $\begin{array}{c}\text { C } \\
\text { ol } \\
\text { or } \\
( \\
\text { L } \\
\text { u) }\end{array}$ & \begin{tabular}{|c}
$\mathrm{Tu}$ \\
rbi \\
dit \\
$\mathrm{y}$ \\
$(\mathrm{N}$. \\
$\mathrm{T}$. \\
$\mathrm{U})$
\end{tabular} & $\begin{array}{l}\text { Tot } \\
\text { al } \\
\text { Ha } \\
\text { rdn } \\
\text { ess } \\
\text { (m } \\
\text { g/l) }\end{array}$ & $\begin{array}{l}\mathrm{C} \\
\mathrm{a} \\
( \\
\mathrm{m} \\
\mathrm{g} / \\
\mathrm{l})\end{array}$ & $\begin{array}{c}\mathrm{M} \\
\mathrm{g} \\
( \\
\mathrm{m} \\
\mathrm{g} / \\
\mathrm{l})\end{array}$ & $\begin{array}{c}\mathrm{N} \\
\mathrm{a} \\
( \\
\mathrm{m} \\
\mathrm{g} / \\
\mathrm{l})\end{array}$ & $\begin{array}{l}\mathrm{K} \\
( \\
\mathrm{m} \\
\mathrm{g} / \\
\mathrm{l})\end{array}$ & \begin{tabular}{|c} 
Ch \\
lor \\
ide \\
(m \\
g/l \\
)
\end{tabular} & \begin{tabular}{|c|}
$\mathrm{Su}$ \\
$\mathrm{lph}$ \\
ate \\
(m \\
$\mathrm{g} / \mathrm{l}$ \\
)
\end{tabular} & $\begin{array}{l}\mathrm{Ph} \\
\text { osp } \\
\text { hat } \\
\mathrm{e} \\
(\mathrm{m} \\
\mathrm{g} / \mathrm{l})\end{array}$ & $\begin{array}{l}\mathrm{Ni} \\
\mathrm{tr} \\
\text { at } \\
\mathrm{e} \\
( \\
\mathrm{m} \\
\mathrm{g} / \\
\mathrm{l})\end{array}$ & \begin{tabular}{|c} 
Bic \\
arbo \\
nate \\
(mg \\
/l)
\end{tabular} & $\begin{array}{l}\mathrm{F} \\
\mathrm{e}\end{array}$ & $\begin{array}{l}\mathrm{P} \\
\mathrm{b}\end{array}$ & $\begin{array}{l}\mathrm{M} \\
\mathrm{n}\end{array}$ & $\begin{array}{l}\mathrm{Z} \\
\mathrm{n}\end{array}$ & $\begin{array}{l}\mathrm{C} \\
\mathrm{u}\end{array}$ \\
\hline $\begin{array}{c}\text { S } \\
\text { K } \\
\text { L1 }\end{array}$ & $\begin{array}{l}7 \\
8 \\
8 \\
\end{array}$ & $\begin{array}{l}2 \\
8 . \\
9\end{array}$ & $\begin{array}{l}2 \\
4 \\
3\end{array}$ & $\begin{array}{c}41 \\
2\end{array}$ & 1 & $\begin{array}{l}14 . \\
32\end{array}$ & $\begin{array}{l}83 . \\
12\end{array}$ & $\begin{array}{l}1 \\
6 . \\
2 \\
1 \\
\end{array}$ & $\begin{array}{l}5 . \\
1 \\
2\end{array}$ & $\begin{array}{c}6 . \\
7 \\
1\end{array}$ & $\begin{array}{l}0 . \\
0 \\
4\end{array}$ & $\begin{array}{l}90 . \\
21\end{array}$ & $\begin{array}{c}2.9 \\
8\end{array}$ & $\begin{array}{c}0.5 \\
2\end{array}$ & $\begin{array}{l}0 . \\
02\end{array}$ & $\begin{array}{c}49.2 \\
2\end{array}$ & $\begin{array}{l}0 \\
9 \\
\end{array}$ & $\begin{array}{l}\dot{0} \\
1 \\
\end{array}$ & $\begin{array}{l}0 \\
0 \\
0 \\
2\end{array}$ & \begin{tabular}{|c|}
0 \\
0 \\
0 \\
\end{tabular} & $\begin{array}{l}0 \\
1 \\
\end{array}$ \\
\hline $\begin{array}{c}\mathrm{S} \\
\mathrm{K} \\
\mathrm{L} 2\end{array}$ & $\begin{array}{l}8 \\
4 \\
3 \\
\end{array}$ & $\begin{array}{l}2 \\
8 . \\
8\end{array}$ & $\begin{array}{l}2 \\
5 \\
6\end{array}$ & $\begin{array}{c}31 \\
2\end{array}$ & 1 & $\begin{array}{l}12 . \\
42\end{array}$ & $\begin{array}{l}97 . \\
36\end{array}$ & $\begin{array}{l}7 . \\
4 \\
3\end{array}$ & $\begin{array}{l}6 . \\
1 \\
0\end{array}$ & $\begin{array}{l}6, \\
7 \\
9\end{array}$ & $\begin{array}{c}0 . \\
1 \\
2\end{array}$ & $\begin{array}{l}89 . \\
32\end{array}$ & $\begin{array}{c}1.9 \\
2\end{array}$ & $\begin{array}{c}0.4 \\
7\end{array}$ & $\begin{array}{c}0 . \\
01\end{array}$ & \begin{tabular}{|c}
50.2 \\
8
\end{tabular} & $\begin{array}{l}1 \\
2\end{array}$ & $\begin{array}{l}\dot{0} \\
1\end{array}$ & $\begin{array}{l}0 \\
0 \\
0 \\
5\end{array}$ & \begin{tabular}{|l}
0 \\
0 \\
0 \\
\end{tabular} & $\begin{array}{l}0 \\
2 \\
\end{array}$ \\
\hline $\begin{array}{c}\mathrm{S} \\
\mathrm{K} \\
\mathrm{L} 3\end{array}$ & $\begin{array}{l}9 \\
4 \\
\end{array}$ & $\begin{array}{c}2 \\
9 . \\
1\end{array}$ & $\begin{array}{l}1 \\
9 \\
8\end{array}$ & $\begin{array}{c}24 \\
6\end{array}$ & 0 & $\begin{array}{l}11 . \\
34\end{array}$ & $\begin{array}{c}12 \\
0.4 \\
3\end{array}$ & $\begin{array}{l}8 . \\
2 \\
1\end{array}$ & $\begin{array}{l}3 . \\
9 \\
7\end{array}$ & $\begin{array}{c}7 . \\
2 \\
1\end{array}$ & $\begin{array}{l}0 . \\
0 \\
6\end{array}$ & $\begin{array}{c}49 . \\
99\end{array}$ & $\begin{array}{c}1.6 \\
3\end{array}$ & $\begin{array}{c}1.0 \\
1\end{array}$ & $\begin{array}{c}0 . \\
05\end{array}$ & $\begin{array}{c}38.1 \\
9\end{array}$ & $\begin{array}{l}1 \\
2 \\
\end{array}$ & $\begin{array}{l}0 \\
2 \\
\end{array}$ & $\begin{array}{l}\dot{0} \\
3\end{array}$ & $\begin{array}{l}\dot{0} \\
4 \\
\end{array}$ & $\begin{array}{l}0 \\
1 \\
\end{array}$ \\
\hline $\begin{array}{c}\text { S } \\
\text { K } \\
\text { L4 }\end{array}$ & $\begin{array}{l}3 \\
2\end{array}$ & $\begin{array}{l}2 \\
9 . \\
7\end{array}$ & $\begin{array}{l}2 \\
6 \\
3\end{array}$ & $\begin{array}{c}41 \\
5\end{array}$ & 0 & $\begin{array}{c}8.0 \\
1\end{array}$ & $\begin{array}{c}14 \\
7.3 \\
4\end{array}$ & $\begin{array}{l}1 \\
2 . \\
3 \\
1\end{array}$ & $\begin{array}{l}4 . \\
1 \\
3\end{array}$ & $\begin{array}{l}8 . \\
3 \\
4\end{array}$ & $\begin{array}{l}0 . \\
1 \\
5\end{array}$ & $\begin{array}{c}38 . \\
78\end{array}$ & $\begin{array}{c}1.8 \\
9\end{array}$ & $\begin{array}{c}0.3 \\
9\end{array}$ & $\begin{array}{c}0 . \\
05\end{array}$ & \begin{tabular}{|c}
39.1 \\
2
\end{tabular} & $\begin{array}{l}0 \\
7\end{array}$ & $\begin{array}{l}0 \\
1\end{array}$ & $\begin{array}{l}\dot{0} \\
0 \\
1\end{array}$ & $\begin{array}{l}\dot{0} \\
5\end{array}$ & $\begin{array}{l}0 \\
1\end{array}$ \\
\hline $\begin{array}{c}\text { S } \\
\text { K } \\
\text { L4 }\end{array}$ & $\begin{array}{l}\dot{5} \\
5\end{array}$ & $\begin{array}{l}2 \\
9 . \\
3\end{array}$ & $\begin{array}{l}1 \\
9 \\
7\end{array}$ & $\begin{array}{c}29 \\
9\end{array}$ & 0 & $\begin{array}{c}7.3 \\
9\end{array}$ & $\begin{array}{c}13 \\
9.3 \\
6\end{array}$ & $\begin{array}{l}1 \\
4 . \\
1 \\
5\end{array}$ & $\begin{array}{l}2 . \\
9 \\
9\end{array}$ & $\begin{array}{l}3 . \\
9 \\
8\end{array}$ & $\begin{array}{c}0 . \\
1 \\
7\end{array}$ & $\begin{array}{l}39 . \\
88\end{array}$ & $\begin{array}{c}1.5 \\
9\end{array}$ & $\begin{array}{c}0.2 \\
9\end{array}$ & $\begin{array}{c}0 . \\
04\end{array}$ & \begin{tabular}{|c|}
49.4 \\
8
\end{tabular} & $\begin{array}{l}1 \\
4\end{array}$ & $\begin{array}{l}\dot{0} \\
1\end{array}$ & $\begin{array}{l}0 \\
\dot{0} \\
2\end{array}$ & $\begin{array}{c}0 \\
. \\
0 \\
8\end{array}$ & $\begin{array}{l}0 \\
1\end{array}$ \\
\hline $\begin{array}{c}\mathrm{S} \\
\mathrm{K} \\
\mathrm{L} 5\end{array}$ & $\begin{array}{l}7 \\
6 \\
6 \\
\end{array}$ & $\begin{array}{l}2 \\
9 . \\
2\end{array}$ & $\begin{array}{l}3 \\
7 \\
5\end{array}$ & $\begin{array}{c}36 \\
7\end{array}$ & 1 & $\begin{array}{c}5.2 \\
3\end{array}$ & $\begin{array}{c}13 \\
1.1 \\
1\end{array}$ & $\begin{array}{c}1 \\
6 . \\
1 \\
1 \\
\end{array}$ & $\begin{array}{l}5 . \\
1 \\
2\end{array}$ & $\begin{array}{l}2 . \\
9 \\
9\end{array}$ & $\begin{array}{c}0 . \\
0 \\
6\end{array}$ & $\begin{array}{l}58 . \\
28\end{array}$ & $\begin{array}{c}5.0 \\
2\end{array}$ & $\begin{array}{c}0.8 \\
9\end{array}$ & $\begin{array}{c}0 . \\
02\end{array}$ & $\begin{array}{c}59.3 \\
9\end{array}$ & $\begin{array}{l}1 \\
6 \\
\end{array}$ & $\begin{array}{l}0 \\
0 \\
0 \\
1\end{array}$ & $\begin{array}{l}0 \\
0 \\
0 \\
1\end{array}$ & \begin{tabular}{|c|}
0 \\
. \\
0 \\
6 \\
\end{tabular} & $\begin{array}{l}0 \\
2 \\
\end{array}$ \\
\hline $\begin{array}{c}\text { S } \\
\text { K } \\
\text { L6 }\end{array}$ & $\begin{array}{l}7 \\
6 \\
6 \\
3\end{array}$ & $\begin{array}{c}2 \\
8 . \\
3\end{array}$ & $\begin{array}{l}2 \\
7 \\
3\end{array}$ & $\begin{array}{c}42 \\
4\end{array}$ & 1 & $\begin{array}{c}4.9 \\
1\end{array}$ & $\begin{array}{l}87 . \\
23\end{array}$ & $\begin{array}{l}1 \\
9 . \\
3 \\
2\end{array}$ & $\begin{array}{l}4 . \\
1 \\
5\end{array}$ & $\begin{array}{l}4 . \\
7 \\
8\end{array}$ & $\begin{array}{l}0 . \\
0 \\
8\end{array}$ & $\begin{array}{c}59 . \\
99\end{array}$ & $\begin{array}{c}2.2 \\
3\end{array}$ & $\begin{array}{c}0.4 \\
9\end{array}$ & $\begin{array}{c}0 . \\
01\end{array}$ & $\begin{array}{c}67.1 \\
2\end{array}$ & $\begin{array}{l}1 \\
2\end{array}$ & $\begin{array}{l}\dot{0} \\
1\end{array}$ & $\begin{array}{l}\dot{0} \\
5\end{array}$ & $\begin{array}{l}\dot{0} \\
7\end{array}$ & $\begin{array}{l}0 \\
1\end{array}$ \\
\hline $\begin{array}{c}\text { S } \\
\text { K } \\
\text { L7 }\end{array}$ & $\begin{array}{l}2 \\
1 \\
\end{array}$ & $\begin{array}{l}2 \\
8 . \\
8\end{array}$ & $\begin{array}{l}2 \\
9 \\
8\end{array}$ & $\begin{array}{c}38 \\
8\end{array}$ & 1 & $\begin{array}{c}7.2 \\
1\end{array}$ & $\begin{array}{l}65 . \\
43\end{array}$ & $\begin{array}{l}1 \\
7 . \\
3 \\
8 \\
\end{array}$ & $\begin{array}{l}3 . \\
8 \\
3\end{array}$ & $\begin{array}{l}7 \\
3 \\
2\end{array}$ & $\begin{array}{l}0 . \\
1 \\
2\end{array}$ & $\begin{array}{l}67 . \\
28\end{array}$ & $\begin{array}{c}1.9 \\
8\end{array}$ & $\begin{array}{c}0.2 \\
3\end{array}$ & $\begin{array}{c}0 . \\
01\end{array}$ & \begin{tabular}{|c|}
73.0 \\
1
\end{tabular} & $\begin{array}{l}0 \\
7 \\
\end{array}$ & $\begin{array}{l}0 \\
2 \\
\end{array}$ & $\begin{array}{l}0 \\
4 \\
\end{array}$ & $\begin{array}{l}0 \\
8 \\
\end{array}$ & $\begin{array}{l}0 \\
2 \\
\end{array}$ \\
\hline S & 8 & 2 & 3 & 41 & 0 & 3.6 & 78. & 1 & 4. & 1. & 0. & 78. & 1.9 & 0.5 & 0. & 28.1 & 0 & 0 & 0 & 0 & 0 \\
\hline
\end{tabular}




\begin{tabular}{|c|c|c|c|c|c|c|c|c|c|c|c|c|c|c|c|c|c|c|c|c|c|}
\hline $\begin{array}{c}\text { K } \\
\text { L8 }\end{array}$ & 5 & $\begin{array}{l}9 . \\
6\end{array}$ & $\begin{array}{l}1 \\
5\end{array}$ & 8 & & 1 & 39 & $\begin{array}{l}4 . \\
2 \\
9\end{array}$ & $\begin{array}{l}6 \\
4\end{array}$ & $\begin{array}{l}9 \\
9\end{array}$ & $\begin{array}{l}1 \\
5\end{array}$ & 12 & 2 & 8 & 03 & 9 & $\begin{array}{l}0 \\
9\end{array}$ & $\begin{array}{l}0 \\
1\end{array}$ & $\begin{array}{l}0 \\
5\end{array}$ & $\begin{array}{l}0 \\
9\end{array}$ & $\begin{array}{l}0 \\
2\end{array}$ \\
\hline $\begin{array}{c}\text { S } \\
\text { K } \\
\text { L9 }\end{array}$ & $\begin{array}{l}8 \\
7 \\
7 \\
1\end{array}$ & $\begin{array}{l}2 \\
9 . \\
1\end{array}$ & $\begin{array}{l}2 \\
9 \\
9\end{array}$ & $\begin{array}{c}38 \\
7\end{array}$ & 1 & $\begin{array}{c}3.9 \\
8\end{array}$ & $\begin{array}{c}85 . \\
55\end{array}$ & $\begin{array}{l}9 . \\
7 \\
5\end{array}$ & $\begin{array}{l}2 . \\
7 \\
3\end{array}$ & $\begin{array}{c}4 . \\
8 \\
2\end{array}$ & $\begin{array}{l}0 . \\
1 \\
8\end{array}$ & $\begin{array}{l}71 . \\
23\end{array}$ & $\begin{array}{c}1.8 \\
2\end{array}$ & $\begin{array}{c}0.2 \\
9\end{array}$ & $\begin{array}{c}0 . \\
06\end{array}$ & $\begin{array}{c}42.3 \\
9\end{array}$ & $\begin{array}{l}\dot{0} \\
6\end{array}$ & $\begin{array}{l}\dot{0} \\
1\end{array}$ & $\begin{array}{l}0 \\
0 \\
0 \\
1\end{array}$ & $\begin{array}{l}0 \\
0 \\
0 \\
3\end{array}$ & \begin{tabular}{|l}
0 \\
0 \\
0 \\
\end{tabular} \\
\hline $\begin{array}{c}\text { S } \\
\text { K } \\
\text { L1 } \\
0\end{array}$ & $\begin{array}{l}8 \\
\dot{9} \\
2\end{array}$ & $\begin{array}{l}2 \\
8 . \\
7\end{array}$ & $\begin{array}{l}3 \\
2 \\
7\end{array}$ & $\begin{array}{c}39 \\
9\end{array}$ & 0 & $\begin{array}{c}4.7 \\
1\end{array}$ & $\begin{array}{l}89 . \\
20\end{array}$ & $\begin{array}{l}6 . \\
7 \\
1\end{array}$ & $\begin{array}{l}1 . \\
9 \\
9\end{array}$ & $\begin{array}{c}4 . \\
9 \\
9\end{array}$ & $\begin{array}{c}0 . \\
1 \\
1\end{array}$ & $\begin{array}{l}88 . \\
02\end{array}$ & $\begin{array}{c}1.3 \\
8\end{array}$ & $\begin{array}{c}0.8 \\
4\end{array}$ & $\begin{array}{c}0 . \\
05\end{array}$ & $\begin{array}{c}38.2 \\
9\end{array}$ & $\begin{array}{l}0 \\
1 \\
1\end{array}$ & $\begin{array}{l}0 \\
. \\
0 \\
2\end{array}$ & $\begin{array}{l}0 \\
. \\
0 \\
1\end{array}$ & $\begin{array}{l}0 \\
. \\
0 \\
2\end{array}$ & \begin{tabular}{|l}
0 \\
0 \\
0 \\
\end{tabular} \\
\hline $\begin{array}{c}\mathrm{S} \\
\mathrm{K} \\
\mathrm{L} 1 \\
1\end{array}$ & $\begin{array}{l}8 \\
. \\
4 \\
5 \\
\end{array}$ & $\begin{array}{l}2 \\
9 . \\
7\end{array}$ & $\begin{array}{l}2 \\
7 \\
9\end{array}$ & $\begin{array}{c}43 \\
9\end{array}$ & 0 & $\begin{array}{c}5.1 \\
2\end{array}$ & $\begin{array}{c}13 \\
2.2 \\
1\end{array}$ & $\begin{array}{l}8 . \\
9 \\
3\end{array}$ & $\begin{array}{l}2 . \\
4 \\
8\end{array}$ & $\begin{array}{c}6 . \\
0 \\
9\end{array}$ & $\begin{array}{l}0 . \\
0 \\
9\end{array}$ & $\begin{array}{l}87 . \\
98\end{array}$ & $\begin{array}{c}1.0 \\
5\end{array}$ & $\begin{array}{c}0.3 \\
9\end{array}$ & $\begin{array}{c}0 . \\
07\end{array}$ & $\begin{array}{c}59.1 \\
5\end{array}$ & $\begin{array}{l}\dot{1} \\
5\end{array}$ & $\begin{array}{l}\dot{0} \\
1\end{array}$ & $\begin{array}{l}\dot{0} \\
2\end{array}$ & $\begin{array}{l}0 \\
. \\
0 \\
4\end{array}$ & \begin{tabular}{|l}
0 \\
0 \\
0 \\
\end{tabular} \\
\hline $\begin{array}{c}\text { S } \\
\text { K } \\
\text { L1 } \\
2\end{array}$ & $\begin{array}{l}8 \\
\dot{1} \\
3\end{array}$ & $\begin{array}{c}3 \\
0 . \\
1\end{array}$ & $\begin{array}{l}2 \\
6 \\
8\end{array}$ & $\begin{array}{c}28 \\
8\end{array}$ & 1 & $\begin{array}{c}7.2 \\
8\end{array}$ & $\begin{array}{c}15 \\
5.2 \\
3\end{array}$ & $\begin{array}{l}9 . \\
8 \\
8\end{array}$ & $\begin{array}{l}3 . \\
5 \\
5\end{array}$ & $\begin{array}{l}7 . \\
8 \\
8\end{array}$ & $\begin{array}{c}0 . \\
0 \\
8\end{array}$ & $\begin{array}{l}69 . \\
44\end{array}$ & $\begin{array}{c}1.2 \\
8\end{array}$ & $\begin{array}{c}0.8 \\
2\end{array}$ & $\begin{array}{c}0 . \\
01\end{array}$ & $\begin{array}{c}39.3 \\
7\end{array}$ & $\begin{array}{l}0 \\
8\end{array}$ & $\begin{array}{l}0 \\
1\end{array}$ & $\begin{array}{l}\dot{0} \\
3\end{array}$ & $\begin{array}{l}\dot{0} \\
6\end{array}$ & \begin{tabular}{|l}
0 \\
0 \\
1
\end{tabular} \\
\hline $\begin{array}{c}\text { S } \\
\text { K } \\
\text { L1 } \\
3\end{array}$ & $\begin{array}{l}8 \\
\dot{3} \\
7\end{array}$ & $\begin{array}{l}2 \\
9 . \\
5\end{array}$ & $\begin{array}{l}1 \\
9 \\
6\end{array}$ & $\begin{array}{c}37 \\
4\end{array}$ & 0 & $\begin{array}{c}8.2 \\
9\end{array}$ & $\begin{array}{c}14 \\
8.3 \\
9\end{array}$ & $\begin{array}{l}1 \\
3 . \\
8 \\
3\end{array}$ & $\begin{array}{l}4 . \\
3 \\
8\end{array}$ & $\begin{array}{c}4 . \\
9 \\
3\end{array}$ & $\begin{array}{l}0 . \\
0 \\
7\end{array}$ & $\begin{array}{c}58 . \\
79\end{array}$ & $\begin{array}{c}1.8 \\
3\end{array}$ & $\begin{array}{c}0.4 \\
8\end{array}$ & $\begin{array}{c}0 . \\
03\end{array}$ & $\begin{array}{c}52.3 \\
7\end{array}$ & \begin{tabular}{|l}
0 \\
0 \\
1 \\
4
\end{tabular} & $\begin{array}{l}0 \\
\dot{0} \\
2\end{array}$ & $\begin{array}{l}0 \\
\dot{0} \\
4\end{array}$ & $\begin{array}{l}0 \\
\dot{0} \\
7\end{array}$ & \begin{tabular}{|l}
0 \\
0 \\
0 \\
2
\end{tabular} \\
\hline $\begin{array}{c}\text { S } \\
\text { K } \\
\text { L1 } \\
4\end{array}$ & $\begin{array}{l}7 \\
\text {, } \\
3 \\
3\end{array}$ & $\begin{array}{l}2 \\
8 . \\
6\end{array}$ & $\begin{array}{l}2 \\
3 \\
1\end{array}$ & $\begin{array}{c}42 \\
4\end{array}$ & 1 & $\begin{array}{c}4.3 \\
7\end{array}$ & $\begin{array}{c}15 \\
3.2 \\
4\end{array}$ & $\begin{array}{l}1 \\
5 . \\
9 \\
9\end{array}$ & $\begin{array}{c}2 . \\
9 \\
7\end{array}$ & $\begin{array}{l}7 . \\
7 \\
1\end{array}$ & $\begin{array}{l}0 . \\
0 \\
9\end{array}$ & $\begin{array}{c}59 . \\
91\end{array}$ & $\begin{array}{c}1.4 \\
8\end{array}$ & $\begin{array}{c}0.3 \\
7\end{array}$ & $\begin{array}{c}0 . \\
07\end{array}$ & $\begin{array}{c}62.0 \\
1\end{array}$ & $\begin{array}{l}0 \\
1 \\
3\end{array}$ & $\begin{array}{l}0 \\
\dot{0} \\
1\end{array}$ & $\begin{array}{l}0 \\
. \\
0 \\
5\end{array}$ & $\begin{array}{l}0 \\
. \\
0 \\
8\end{array}$ & \begin{tabular}{|l}
0 \\
0 \\
0
\end{tabular} \\
\hline $\begin{array}{c}\mathrm{S} \\
\mathrm{K} \\
\mathrm{L} 1 \\
5\end{array}$ & $\begin{array}{l}8 \\
2 \\
2 \\
4\end{array}$ & $\begin{array}{l}2 \\
9 . \\
6\end{array}$ & $\begin{array}{l}3 \\
5 \\
7\end{array}$ & $\begin{array}{c}36 \\
6\end{array}$ & 1 & $\begin{array}{c}2.3 \\
1\end{array}$ & $\begin{array}{c}13 \\
9.4 \\
6\end{array}$ & $\begin{array}{l}1 \\
9 . \\
3 \\
2\end{array}$ & $\begin{array}{l}3 . \\
8 \\
8\end{array}$ & $\begin{array}{c}3 . \\
9 \\
6\end{array}$ & $\begin{array}{l}0 . \\
0 \\
2\end{array}$ & $\begin{array}{l}61 . \\
19\end{array}$ & $\begin{array}{c}2.1 \\
1\end{array}$ & $\begin{array}{c}0.2 \\
6\end{array}$ & $\begin{array}{c}0 . \\
07\end{array}$ & $\begin{array}{c}72.3 \\
1\end{array}$ & $\begin{array}{l}0 \\
\text { i } \\
7\end{array}$ & $\begin{array}{l}0 \\
0 \\
0 \\
1\end{array}$ & $\begin{array}{l}0 \\
\dot{0} \\
1\end{array}$ & $\begin{array}{l}0 \\
\dot{0} \\
2\end{array}$ & \begin{tabular}{|l} 
\\
0 \\
0 \\
1
\end{tabular} \\
\hline $\begin{array}{c}\text { S } \\
\text { K } \\
\text { L1 } \\
6\end{array}$ & $\begin{array}{l}7 \\
\dot{5} \\
2\end{array}$ & $\begin{array}{l}2 \\
9 . \\
3\end{array}$ & $\begin{array}{l}3 \\
2 \\
1\end{array}$ & $\begin{array}{c}43 \\
1\end{array}$ & 1 & $\begin{array}{c}1.9 \\
8\end{array}$ & $\begin{array}{c}12 \\
3.2 \\
1\end{array}$ & $\begin{array}{l}2 \\
0 . \\
0 \\
1\end{array}$ & $\begin{array}{l}2 . \\
6 \\
2\end{array}$ & $\begin{array}{l}6 . \\
3 \\
2\end{array}$ & $\begin{array}{l}0 . \\
0 \\
1\end{array}$ & $\begin{array}{c}74 . \\
59\end{array}$ & $\begin{array}{c}1.0 \\
3\end{array}$ & $\begin{array}{c}0.1 \\
8\end{array}$ & $\begin{array}{c}0 . \\
01\end{array}$ & $\begin{array}{c}81.0 \\
1\end{array}$ & $\begin{array}{l}0 \\
. \\
0 \\
9\end{array}$ & $\begin{array}{l}0 \\
\dot{0} \\
1\end{array}$ & $\begin{array}{l}0 \\
. \\
0 \\
5\end{array}$ & $\begin{array}{l}0 \\
. \\
0 \\
5\end{array}$ & \begin{tabular}{|l}
0 \\
0 \\
0 \\
1
\end{tabular} \\
\hline $\begin{array}{c}\text { S } \\
\text { K } \\
\text { L1 } \\
7\end{array}$ & $\begin{array}{l}8 \\
8 \\
8 \\
5\end{array}$ & $\begin{array}{l}2 \\
8 . \\
7\end{array}$ & $\begin{array}{l}3 \\
4 \\
7\end{array}$ & $\begin{array}{c}28 \\
8\end{array}$ & 0 & $\begin{array}{c}6.8 \\
7\end{array}$ & $\begin{array}{l}89 . \\
56 .\end{array}$ & $\begin{array}{c}1 \\
9 . \\
8 \\
8\end{array}$ & $\begin{array}{l}3 . \\
8 \\
3\end{array}$ & $\begin{array}{c}7 . \\
9 \\
3\end{array}$ & $\begin{array}{l}0 . \\
0 \\
5\end{array}$ & $\begin{array}{l}81 . \\
34\end{array}$ & $\begin{array}{c}1.4 \\
7\end{array}$ & $\begin{array}{c}0.9 \\
8\end{array}$ & $\begin{array}{c}0 . \\
04\end{array}$ & $\begin{array}{c}71.0 \\
7\end{array}$ & $\begin{array}{l}0 \\
\dot{1} \\
2\end{array}$ & $\begin{array}{l}0 \\
. \\
0 \\
2\end{array}$ & $\begin{array}{l}0 \\
0 \\
0 \\
6\end{array}$ & $\begin{array}{l}0 \\
. \\
0 \\
3\end{array}$ & \begin{tabular}{|l}
0 \\
0 \\
1
\end{tabular} \\
\hline $\begin{array}{c}\mathrm{S} \\
\mathrm{K} \\
\mathrm{L} 1 \\
8\end{array}$ & $\begin{array}{l}8 \\
\dot{4} \\
9\end{array}$ & $\begin{array}{l}2 \\
9 . \\
5\end{array}$ & $\begin{array}{l}2 \\
8 \\
3\end{array}$ & $\begin{array}{c}32 \\
4\end{array}$ & 0 & $\begin{array}{l}12 . \\
37\end{array}$ & $\begin{array}{l}78 . \\
99\end{array}$ & $\begin{array}{l}1 \\
5 . \\
7 \\
3\end{array}$ & $\begin{array}{l}4 . \\
0 \\
2\end{array}$ & $\begin{array}{l}5 . \\
8 \\
6\end{array}$ & $\begin{array}{l}0 . \\
0 \\
7\end{array}$ & $\begin{array}{c}82 . \\
99\end{array}$ & $\begin{array}{c}1.1 \\
7\end{array}$ & $\begin{array}{c}0.3 \\
7\end{array}$ & $\begin{array}{c}0 . \\
08\end{array}$ & $\begin{array}{c}84.0 \\
1\end{array}$ & $\begin{array}{l}1 \\
5\end{array}$ & $\begin{array}{l}0 \\
. \\
0 \\
1\end{array}$ & $\begin{array}{l}0 \\
0 \\
2\end{array}$ & $\begin{array}{l}0 \\
. \\
0 \\
4\end{array}$ & \begin{tabular}{|l}
0 \\
0 \\
0
\end{tabular} \\
\hline$S$ & 8 & 2 & 1 & 31 & 0 & 11. & 13 & 1 & 3. & 9. & 0. & 69. & 1.0 & 0.5 & 0. & 61.0 & 0 & 0 & 0 & 0 & 0 \\
\hline
\end{tabular}




\begin{tabular}{|c|c|c|c|c|c|c|c|c|c|c|c|c|c|c|c|c|c|c|c|c|c|}
\hline $\mathrm{K}$ &. & 8. & 8 & 1 & & 34 & 2.1 & 4. & 8 & 0 & 0 & 49 & 2 & 9 & 01 & 1 &. &. &. &. &. \\
$\mathrm{~L} 1$ & 5 & 7 & 5 & & & & 4 & 9 & 8 & 1 & 8 & & & & & & 0 & 0 & 0 & 0 & 0 \\
9 & 1 & & & & & & & 9 & & & & & & & & & 9 & 1 & 1 & 6 & 2 \\
\hline $\mathrm{S}$ & 7 & 3 & 2 & 26 & 1 & 15. & 16 & 1 & 2. & 6 & 0. & 49. & 1.0 & 0.7 & 0. & 39.1 & 0 & 0 & 0 & 0 & 0 \\
$\mathrm{~K}$ &. & 0. & 1 & 9 & & 21 & 4.3 & 0. & 6 & 3 & 0 & 43 & 6 & 9 & 05 & 8 &. &. &. &. &. \\
$\mathrm{~L} 2$ & 8 & 2 & 1 & & & & 4 & 1 & 7 & 9 & 9 & & & & & & 0 & 0 & 0 & 0 & 0 \\
0 & 8 & & & & & & & 7 & & & & & & & & & 8 & 2 & 3 & 3 & 1 \\
\hline
\end{tabular}

Table 2: Comparison of obtained values with the World Health Organization (WHO) standard

\begin{tabular}{|c|c|c|c|c|}
\hline Parameter & $\begin{array}{l}\text { Highest } \\
\text { desirable } \\
(\mathrm{mg} / \mathrm{l}) \\
\end{array}$ & $\begin{array}{c}\text { Maximum } \\
\text { permissible level } \\
(\mathrm{mg} / \mathrm{l})\end{array}$ & $\begin{array}{c}\text { Range of values } \\
\text { obtained from } \\
\text { analysis (mg/l) }\end{array}$ & Remarks \\
\hline $\mathrm{Ph}$ & $7.0-8.9$ & $6.5-9.5$ & 6.94-8.92 & It is neutral to alkaline \\
\hline Conductivity & $900(\mathrm{~S} / \mathrm{m})$ & $1200(\mathrm{~S} / \mathrm{m})$ & $246-439$ & Extremely low \\
\hline Total hardness & 100 & 100 & $65.43-164.34$ & $\begin{array}{l}\text { Moderately to very hard } \\
\text { water }\end{array}$ \\
\hline Magnesium & 20 & 20 & $1.99-20.01$ & Extremely low \\
\hline Calcium & 0.01 & 0.07 & $6.71-20.01$ & Highly concentrated \\
\hline Sulphate & 250 & 500 & $1.02-5.02$ & Suitable for drinking \\
\hline Iron & 1.0 & 3.0 & $0.08-0.16$ & ok \\
\hline Nitrate & 10 & 50 & $0.01-0.08$ & ok \\
\hline Lead & 0.01 & 0.01 & $0.01-0.01$ & ok \\
\hline Turbidity & 5.0 & 5.0 & $1.16-15.32$ & $\begin{array}{c}18 \text { samples out of } 20 \text { are } \\
\text { not suitable }\end{array}$ \\
\hline Sodium & - & 200 & $1.72-8.34$ & Suitable for drinking \\
\hline Manganese & 0.0 & 0.4 & $0.01-0.05$ & Suitable for drinking \\
\hline Zinc & 0.01 & 3.0 & $0.01-0.08$ & Suitable for drinking \\
\hline Copper & 0.05 & 2.0 & $0.01-0.02$ & Suitable for drinking \\
\hline $\begin{array}{l}\text { Total Organic } \\
\text { carbon }\end{array}$ & - & 5 & $0.01-0.07$ & ok \\
\hline $\begin{array}{l}\text { Total dissolved } \\
\text { solids (mg/l) }\end{array}$ & & 500 & $90-366$ & ok \\
\hline Potassium & 10.0 & 11.0 & $0.02-0.18$ & ok \\
\hline
\end{tabular}

The variables determined are: $\mathrm{pH}$ ranges from $7.67-8.56 \mathrm{mg} / \mathrm{l}$ which is suggestive of neutral to alkaline in character, calcium ranges from $5.12-24.16 \mathrm{mg} / \mathrm{l}$, turbidity ranges from $1.16-15.32 \mathrm{mg} / \mathrm{l}$, total dissolved solid (Tds) ranges from $90-366 \mathrm{mg} / \mathrm{l}$ and total hardness ranges from 58.65-187.37 mg/l. They are suggestive of concentration of detergent from soap, calcium, magnesium, suspended solid particles and colloidal matters from some of the water samples. While iron ranges from 0.08-0.16 mg/l, potassium ranges from $0.02-0.18 \mathrm{mg} / \mathrm{l}$, chloride ranges from 30.03-120.13 mg/l, sulphate ranges from 1.03-5.36 mg/l, nitrate ranges from 0.01-0.23 mg/l, lead ranges from 0-0.01 mg/l, Zinc ranges from 0$0.08 \mathrm{mg} / \mathrm{l}$, copper ranges from $0-0.02 \mathrm{mg} / \mathrm{l}$ and magnesium ranges from $1.38-6.56 \mathrm{mg} / \mathrm{l}$, fall within standards. Coliform count ranges from $0-14 \mathrm{mg} / \mathrm{l}$. The water should be treated before the consumption because of its high concentration of detergent, suspended particles, faecal materials and calcium from the water samples. The quarry pits should be reclaimed and rehabilitate after mining. Alkaline materials 
should be used to neutralize the rock pile area, dumped site; tailing and mine pit itself to avoid acid generation.

Table 3: water classification according to its hardness [11]

\begin{tabular}{|c|c|}
\hline Hardness & Types of hardness \\
\hline $0-60$ & Soft water \\
\hline $61-120$ & Moderately hard water \\
\hline $121-180$ & Hard water \\
\hline$>180$ & Very hard water \\
\hline
\end{tabular}

The result shows that turbidity, hardness, TDS, magnesium and calcium are very high in some samples. That calcium had the greatest values both for fresh water and drinking water respectively. That the environment is more of calcite than dolomite make it calcitic dolomite environment. From the potential health effects like urinary tract infection, bacteraemia and diarrhoea from the injection of water containing such concentration above mentioned parameters, the water collected should be treated before consumption. The color of the samples collected, ranges from 0-1 hazen units were not all cleared as a result of high concentration of dissolved calcium and magnesium. The $\mathrm{pH}$ of surface and groundwater in the study area generally ranges from 7.61-8.65, which means that the $\mathrm{pH}$ is generally neutral to alkaline. Electrical conductivity generally ranges from 182.1-733 $(\mu \mathrm{S} / \mathrm{m})$, which means the sample are good conductor of electricity.

\subsection{Environmental risk analysis on surface water and underground water caused by marble mining}

\subsubsection{Impact Analysis}

The effects on water quality and availability of water resources in the project area constitute the most important impact.

This section assesses the impacts of marble mining on the quality and quantity of water. This means that our work predicts how much the surface and groundwater reference levels would change as a result of contaminants from the mine.

There are six types of impacts from marble mining that affect the water quality of SakalalinaMadagascar:

- Discharge of pollutants from water from quarrying lake;

- The drainage of mining acid

- Metal contamination and filtration

- Pollution by chemicals

- Blasting and explosive substances

- Erosion of soils and mining waste in surface water

\section{Discharge of pollutants from water from Quarry Lake:}

To analyze pollution from the quarry lake, it is estimated the precipitation and evaporation from the quarry lake surface, runoff from the high quarry walls, groundwater flow from the quarry, flow from any incoming surface water source [22]. According to the study, the oxidation of sulphide minerals in quarry lake walls is also releasing metals and acids into the groundwater. One perceived issue that has 
been raised is that mine lakes could cause an increase in the evapotranspiration (ET) rates that would lower water levels and thus adversely impact sensitive environments on water. The lakes excavated into aquifer rock could allow for easier entry of pollutants or contaminants into the aquifer and ultimately, into the water supply. Public water supply wells are located a minimum $1 \mathrm{Km}$ from mine lakes near both the study area and the transport processes of contaminants and pathogenic organisms from surface-water bodies into and through aquifers has been intensely studied and well documented in the scientific literature.

\section{Mining acid drainage:}

Rocky Acid Drainage (ARD) is a natural process where sulfuric acid is produced when the sulphide in rocks is exposed to air and water. Acid Mining Drainage (DMA) is practically the same process but amplified. When the sulphides in the rock are extracted and exposed to water and air during mining, they form sulfuric acid. This acidic water can dissolve other harmful metals in the surrounding rock. If not controlled, mine acid drainage may flow into streams or rivers or into groundwater. This process can last for hundreds even thousands of years. Acid moves out of mine sites through rainwater or drainage and seeps into streams such as rivers, lakes and groundwater. DMA severely degrades water quality, destroys aquatic life and makes water virtually unusable [23].

\section{Metal contamination and filtration:}

Metal contamination is caused by arsenic, cobalt, copper, cadmium, lead, silver and zinc in rocks when in contact with water. The metals are filtered and the water that moves downstream cleans the top of the rocks. The metals can become stable when the $\mathrm{pH}$ level is neutral. On the other hand, the filtration process is accelerated when the $\mathrm{pH}$ level is low as in acid mine drainage (AMD) [22].

\section{Pollution by chemicals:}

This type of pollution occurs when chemical agents (such as cyanide or sulfuric acids are used to separate the mineral from the ore) spill or filter into nearby bodies of water. These chemicals can be very toxic to the fauna and the flora [15].

\section{Pollution by the use of blasting and blasting substances:}

This section shows the potential impacts of exploitation during the use of explosive and detonating substances on environmental components. Each component of the environment is affected by the use of explosive and detonating substances, which cause impacts on water. Blasting is faster, more efficient and more cost-effective for the company as it can produce up to several thousand tons of rock cut for a single firing flap. The fracking of the massif during the explosion is an element that facilitates the shaking and handling of the rock after collapse since the rock blocks from the shooting parameters, become more and easier to handle [14]. Despite these benefits during operation, this method of slaughter also has negative effects that have a much greater impact on water and that must not be neglected during the firing. Indirect degradation of groundwater could result from blasting which causes a temporary shaking of the rock and results in the new rock fracture near working area of the mine. Blasting can also cause the old pre-existing rock fracture to become more open or permeable, by loosening mineral debris or cement in this fracture; this could affect nearly vertical fractures located up 
to several hundred feet away from the surface mine causing vertical leakage of mine drainage from nearby abandoned deep mines to underlying aquifers [13].

\section{Erosion of soils and mining waste in surface water:}

According to our research, for most mining projects, the potential for erosion of soil and sediment in surface water and the degradation of its quality is a serious problem. Due to the large area of land damaged by marble mining and the large amount of materials that are exposed on site, erosion is a major concern at the site. Erosion causes significant loading of sediments (and any accompanying chemical pollutants) to nearby bodies of water, especially during severe storms and periods of snowmelt [11].

Major sources of erosion and sediment accumulation at the site include storage, heap leaching, rock waste and overburden, tailings piles and dams, transportation routes of materials and roadways access, ore stacks, vehicle and equipment maintenance areas, exploration zones and rehabilitation areas [9].

Table 4: Summary of impact sources of marble mining.

\begin{tabular}{|c|c|}
\hline Sources of impact & Possible impacts \\
\hline $\begin{array}{l}\text { Discharge of pollutants from water } \\
\text { from quarrying lake; }\end{array}$ & $\begin{array}{c}\text { Allow for easier entry of pollutants into the aquifer and public water } \\
\text { Releases metals and acids in groundwater } \\
\text { Risk of contamination of surface waters }\end{array}$ \\
\hline $\begin{array}{l}\text { The drainage of mining acid et la } \\
\text { lixiviation des contaminants }\end{array}$ & $\begin{array}{c}\text { Promotes the formation of sulfuric acid and facilitates the } \\
\text { dissolution of other harmful metals in the surrounding rock } \\
\text { Produces pollution of streams and rivers in the study area } \\
\text { Destroys aquatic life } \\
\text { Makes water almost unusable }\end{array}$ \\
\hline Metal contamination and filtration & $\begin{array}{c}\text { Produce the increases in } \mathrm{pH} \text { and precipitation of } \mathrm{Fe}^{3+} \\
\text { Discoloration and turbidity of water }\end{array}$ \\
\hline Pollution by chemicals & $\begin{array}{c}\text { Reduction of flora and fauna } \\
\text { Reduction of water quality }\end{array}$ \\
\hline blasting and explosive substances & $\begin{array}{c}\text { Facilitates the shaking and handling of rock } \\
\text { Causes pollution of groundwater } \\
\text { Facilitates the dissolution of other metals from the rock } \\
\text { Indirect degradation of groundwater } \\
\text { Causes a temporary shaking of the rock and results in the new } \\
\text { rock fracture near working area of the mine } \\
\text { Causes the old pre-existing rock fracture to become more } \\
\text { open or permeable } \\
\text { Affect nearly vertical fractures located up to several hundred } \\
\text { feet away from the surface mine }\end{array}$ \\
\hline $\begin{array}{l}\text { Erosion of soils and mining waste in } \\
\text { surface water }\end{array}$ & $\begin{array}{c}\text { Causes significant change of sediments (chemical pollutants) to } \\
\text { nearby water bodies } \\
\text { Potential increase in risk of erosion, collapse and landslide. } \\
\text { Risk of landslide } \\
\text { Minimizes of water quality }\end{array}$ \\
\hline
\end{tabular}




\subsubsection{Consequences related to water pollution}

Impairment of the quality of natural waters affects the life of organisms in aquatic systems. Water pollution can alter natural living conditions in a variety of ways (changes in oxygen content, temperature, and water nutrients, direct toxic effects). When released into a river, metal pollutants can accumulate in sediments, plants and aquatic animals [3].

We will see the ecological consequences, the health consequences, the socio-economic consequences:

Table 5: Consequences related to water pollution.

\begin{tabular}{|c|c|}
\hline Type of consequences & Consequences \\
\hline $\begin{array}{l}\text { The consequences caused by nitrates and } \\
\text { phosphates }\end{array}$ & $\begin{array}{c}\text { Pause the phenomenon of eutrophication } \\
\text { Poisoning and degradation of fauna and flora } \\
\text { Causes a high mortality of fish } \\
\text { Danger to public health and the origin of } \\
\text { epidemic diseases } \\
\text { Cause various nuisances }\end{array}$ \\
\hline Ecological consequences & $\begin{array}{c}\text { Causes the propagation of aerobic bacteria } \\
\text { Causes poisoning and degradation of flora and fauna } \\
\text { by toxic }\end{array}$ \\
\hline The health consequences & $\begin{array}{c}\text { Constitutes a major crisis of diarrhea in the } \\
\text { active population } \\
\text { Cause different kinds of diseases (Cholera, } \\
\text { typhoid fever, stomach cancer ...) } \\
\text { Diseases of the skin }\end{array}$ \\
\hline The socio-economic consequences & $\begin{array}{c}\text { Reduction of the number of usable resources } \\
\text { for the Production of drinking water and } \\
\text { increase additional costs of treatment of } \\
\text { potabilisation } \\
\text { The pollution of the water has a great } \\
\text { consequence on the industry, on the } \\
\text { agricultural }\end{array}$ \\
\hline
\end{tabular}

\subsubsection{Risk management measures}

Protection must remain the primary goal of society even if it means reduced production.

There was a lot of problem with water quality and waste management. For our present and future generations, we must safeguard the quality of our water. We must ensure that better prevention strategies in cases where pollution can be avoided.

After identifying the different effects that it could potentially occur during the activity, corresponding mitigation measures are considered and recommended to ensure that the project operates in a more beneficial and less destructive way. 


\section{Risk control measures on the discharge of pollutants from water from quarrying lake:}

The following measures are recommended for the prevention of the release of water pollutants from quarrying lakes: basins to control pollution were built: polluted runoff and dirty rain water will be deposited in these basins and this water will be recycled and used for dust control; drainage channel and water pumps will be introduced inside the pits to collect mining water and transport it to a basin located at the surface; mine lake prevention: the mine lake causes substantial impacts on water in the study area. It is necessary to backfill and recreates its outline and revegetation, to recreate the conditions of pre-exploitation.

\section{General measures on mining acid drainage:}

Precaution to avoid water pollution such as acid mine drainage has been done during the conception of MTH mining project in Sakalalina and the following measures have been taken to avoid the acid mine drainage: limit the exposure of potential acid generator materials by phasing development and construction, with coating, and separating runoff for treatment; implementation of water management techniques such as diversion of clean runoff water from potential acid generator materials and controlled placement of potential acid generator materials (including waste) to permanently create the conditions to avoid contact with oxygen or water; prevent future loss of aquatic life caused by Acid Mining Drainage; listed and cleaned the acid produced by the mine site; improve public access to information and strengthen the control of the treatment of Acid Mining Drainage by MTH society; prevent the Acid Mining Drainage process by better assessing environmental risks and adopting a prevention process.

\section{Measures on metal contamination and filtration / measures on chemical pollution:}

To begin to address the issues related to metal contamination and filtration, we have to: isolate acid leaching generating equipment from oxidation or percolation water, such as tailings ponds with dams and dewatering and subsequent capping. Spreading disposal alternatives should be designed, constructed and operated in accordance with internationally recognized geotechnical safety standards; avoid the infiltration of toxic leachate solutions by the installation of appropriate coatings and underground drainage systems that allow the collection or recycling of these solutions for future treatment, and minimize infiltration into the soil; design the installation of pipelines carrying rich solutions with a confinement bordered by a secondary protection wall; install leak detection equipment in treatment systems with appropriate leak response systems; treatment solution settling ponds and other protective dams designed to retain non-potable water or untreated leachate process effluents should be covered and equipped with sufficient wells to permit level control and water quality. ...; decommissioned leach equipment facilities should use a combination of surface management systems, collection of leaks, and active or passive treatment systems to ensure that the quality of the water resource after shutdown is maintained; establishment of the appropriate drainage system starting from the extraction zone and at the periphery of the various zones of action and existing deposits (dewatering, use of pipes, construction of canals, dike, gutter, ...) to avoid leaks, spills, uncontrolled or uncontrolled infiltration; put the chemicals in containers and paying attention to their overflow. 


\section{Measures on effects by blasting and explosive substances:}

The following measures for the prevention of impacts caused by the use of explosive and detonating substances are recommended: the risk of surface water contamination caused by the use of explosives must be avoided; it is necessary to manage polluting waste surface water; set up of settling basin; comply with all laws and regulations for the use of explosives.

\section{Measures on the impacts of soil erosion and mining waste in surface water:}

Soil and mine waste erosion in surface and groundwater is a serious and adverse environmental consequence in the study area. So we recommend the following measures: domestic wastewater treatment plant for mining employees will be built; reducing the exposure of sediment-generating materials to wind or water (for example, proper placement of soil and rock piles); the diversion of runoff from undisturbed areas around disturbed areas including areas that have been leveled, sown, or planted. Such drainage should be treated for sediment removal; reduction or prevention of off-site sediment transport (eg, use of settling ponds, sludge barriers); rainwater drains, ditches, and stream beds should be protected against erosion by a combination of such factors as: adequate dimensions, slope limiting techniques, and use of riprap and coating; the timely implementation of an appropriate combination of contouring, earthworks, slope reduction / minimization, runoff rate limitation and appropriate drainage facilities to reduce erosion both in areas active and inactive; material access and transportation routes should have gradients or surface treatment to limit erosion, and road drainage systems should be provided; delimit the affected areas to a minimum; storm water settling facilities should be designed and maintained in accordance with internationally accepted good engineering practice, including provisions for the capture of debris and floating material; vegetation of disturbed areas including seeding should be done immediately after application of the substrate to prevent erosion. We try to minimize the clearing, to preserve as much as possible the vegetal cover bordering the zone of action of our activity, to arrange and proceed with the reforestation of the trees with strong growth to the contours of the various specific works to be put in place.

\section{Other measures and monitoring of surface water and groundwater quality:}

Monitoring is one of the most important aspects of a water quality protection program. Drilling is normally required upstream and downstream (in the direction of groundwater flow) to monitor changes in water level and quality over all; comply with all applicable laws and regulations; carry out all the legal obligations that we incumbent on, especially with regard to municipal regulations, and concerning sanitary controls (diseases) and safety (animals, plants and dangerous insects); description of the operation of the sanitary facilities, garbage treatment in the camp; water pollution should be prevented both inside and outside the site. 
Table 6: Follow-up activities of the water management plan.

\begin{tabular}{|c|c|c|c|c|c|c|}
\hline $\begin{array}{c}\text { Sector } \\
\text { component }\end{array}$ & Follow up action & $\begin{array}{l}\text { Follow } \\
\text { up } \\
\text { parameter }\end{array}$ & $\begin{array}{l}\text { Follow up } \\
\text { indicator }\end{array}$ & $\begin{array}{l}\text { Follow-up } \\
\text { program }\end{array}$ & $\begin{array}{c}\text { Source of } \\
\text { control }\end{array}$ & $\begin{array}{c}\text { Frequency } \\
\text { of follow- } \\
\text { up }\end{array}$ \\
\hline $\begin{array}{c}\text { Surface } \\
\text { water and } \\
\text { groundwater }\end{array}$ & $\begin{array}{c}\text { Water } \\
\text { management } \\
\text { (quantitative and } \\
\text { qualitative } \\
\text { management of } \\
\text { water resources): } \\
\text { 1. preservation of } \\
\text { the quality of the } \\
\text { water against } \\
\text { pollution by solid } \\
\text { and liquid waste } \\
\text { resulting from } \\
\text { construction } \\
\text { work } \\
\text { 2. respect of the } \\
\text { load capacity } \\
\text { (pressure) on the } \\
\text { water points } \\
\text { 3. maintenance } \\
\text { of normal water } \\
\text { flow and } \\
\text { preservation of } \\
\text { stormwater } \\
\text { drainage systems } \\
\text { 4. Groundwater } \\
\text { supply } \\
5 \text { protection of } \\
\text { watersheds }\end{array}$ & $\begin{array}{c}\text { Qualities } \\
\text { and } \\
\text { quantities } \\
\text { of water }\end{array}$ & $\begin{array}{l}\text { Numbers } \\
\text { and water } \\
\text { points } \\
\text { frequented } \\
\text { before and } \\
\text { after the } \\
\text { works } \\
\text { Chemical } \\
\text { quality (ph, } \\
\text { phosphorus, } \\
\text { nitrate, etc.) } \\
\text { Ccolor of } \\
\text { water } \\
\text { Biological } \\
\text { properties } \\
\text { of water } \\
\text { Source of } \\
\text { diseases } \\
\text { from water- } \\
\text { flow, water } \\
\text { level }\end{array}$ & $\begin{array}{l}\text { Control of } \\
\text { hydrological } \\
\text { regime of } \\
\text { watercourse } \\
\text { and } \\
\text { groundwater } \\
\text { Control of } \\
\text { the physico- } \\
\text { chemical } \\
\text { and } \\
\text { biological } \\
\text { quality of } \\
\text { watercourses } \\
\text { Knowledge } \\
\text { of the } \\
\text { maximum } \\
\text { capacity of } \\
\text { the aquifer }\end{array}$ & $\begin{array}{c}\text { Sampling } \\
\text { campaign } \\
- \\
\text { investigation } \\
\text { Report of } \\
\text { laboratory } \\
\text { water } \\
\text { analyzes } \\
\text { Technical } \\
\text { study report }\end{array}$ & $\begin{array}{l}\text { During and } \\
\text { after } \\
\text { exploitation }\end{array}$ \\
\hline
\end{tabular}




\subsubsection{Synthesis of the impact study and the various negative impact mitigation measures}

Table 7: Synthesis of sources of impacts and mitigation / compensation measures.

\begin{tabular}{|c|c|c|}
\hline Sources & Possible impacts & Mitigation measures \\
\hline $\begin{array}{c}\text { Pollutants of water from } \\
\text { quarrying lake }\end{array}$ & $\begin{array}{l}\text { Allow for easier entry of pollutants into the } \\
\text { aquifer and public water } \\
\text { Releases metals and acids in groundwater } \\
\text { Risk of contamination of surface waters }\end{array}$ & $\begin{array}{l}\text { Basins to control pollution } \\
\text { were built } \\
\text { Drainage channel and water } \\
\text { pumps will be introduced } \\
\text { inside the pits to collect } \\
\text { mining water and transport it } \\
\text { to a basin located at the } \\
\text { surface }\end{array}$ \\
\hline $\begin{array}{l}\text { Mine acid drainage and } \\
\text { leaching of } \\
\text { contaminants }\end{array}$ & $\begin{array}{l}\text { Promotes the formation of sulfuric acid and } \\
\text { facilitates the dissolution of other harmful } \\
\text { metals in the surrounding rock } \\
\text { Produces pollution of streams and rivers in } \\
\text { the study area } \\
\text { Destroys aquatic life } \\
\text { Makes water almost unusable }\end{array}$ & $\begin{array}{c}\text { Limit the exposure of } \\
\text { chemical materials } \\
\text { Application of water } \\
\text { management techniques } \\
\text { Prevent future loss of aquatic } \\
\text { life } \\
\text { Listed and cleaned the acid } \\
\text { produced by the mine site } \\
\text { Strengthen the control of } \\
\text { Mine acid drainage treatment }\end{array}$ \\
\hline $\begin{array}{l}\text { Chemicals / Metal } \\
\text { Contamination and } \\
\text { Filtration }\end{array}$ & $\begin{array}{l}\text { Produce the increases in } \mathrm{pH} \text { and } \\
\text { precipitation of Fe3+ } \\
\text { Discoloration and turbidity of water } \\
\text { Reduction of flora and fauna } \\
\text { Reduction of water quality }\end{array}$ & $\begin{array}{c}\text { Isolate acid leaching } \\
\text { generating material from } \\
\text { oxidation } \\
\text { Avoid the infiltration of toxic } \\
\text { solutions of leaching } \\
\text { Install leak detection } \\
\text { equipment } \\
\text { Setting up the proper drainage } \\
\text { system }\end{array}$ \\
\hline $\begin{array}{l}\text { Blasting and explosive } \\
\text { substances }\end{array}$ & $\begin{array}{l}\text { Facilitates the shaking and handling of } \\
\text { rock } \\
\text { Causes pollution of groundwater } \\
\text { Facilitates the dissolution of other metals } \\
\text { from the rock }\end{array}$ & $\begin{array}{c}\text { Avoid the risk of } \\
\text { contamination of surface } \\
\text { waters } \\
\text { Manage polluting waste } \\
\text { surface water } \\
\text { Set up of settling basin } \\
\text { Respect all laws and } \\
\text { regulations for the use of } \\
\text { explosives }\end{array}$ \\
\hline $\begin{array}{l}\text { Erosion of soil and } \\
\text { mining waste }\end{array}$ & $\begin{array}{l}\text {-Causes significant change of sediments } \\
\text { (chemical pollutants) to nearby water } \\
\text { bodies } \\
\text { Potential increase in risk of erosion, }\end{array}$ & $\begin{array}{l}\text { Domestic wastewater } \\
\text { treatment plant for mining } \\
\text { employees will be built } \\
\text { Prevention of off-site }\end{array}$ \\
\hline
\end{tabular}




\begin{tabular}{|c|c|c|}
\hline $\begin{array}{c}\text { collapse and slippage } \\
\text { grounds } \\
\text { Risk of landslide- minimise of water } \\
\text { quality }\end{array}$ & $\begin{array}{c}\text { sediment transport } \\
\text { Rainwater drainage pipes, } \\
\text { ditches, and stream beds } \\
\text { should be protected } \\
\text { Delimit the affected areas to a } \\
\text { minimum } \\
\text { Reforestation of disturbed } \\
\text { areas }\end{array}$ \\
\hline
\end{tabular}

\section{Conclusion}

Water pollution is an environmental problem that is of major concern to us in Madagascar. Water pollution has adverse effects on aquatic ecosystems, on living beings. This work was produced as part of the environmental impact study of the marble mining on the water of the SAKALALINAMADAGASCAR area based on environmental risk analyzes on surface water and water underground and risk management measures. We know that Sakalalina's marble exploitation contributes to the promotion of the socio-economic development of the area through the creation of jobs, the reinforcement of infrastructures, the contribution of taxes and mining royalty. But this marble exploitation has negative impacts in the study area, especially at the level of water resources.

The main conclusion that can be taken from this work is more than $60 \%$ of household in Sakalalina use water from unprotected wells and rivers. If ground water and surface water be contaminated, the quality of drinking water will also be bad and the people can suffer. And the People in Sakalalina use water from the river for agriculture and fishing which means that if water resources be contaminated, people living there will suffer to survive. Various environmental measures are proposed in this work such as mitigation measures, avoidance measures, rehabilitation measures and safety measures. These measures are designed to reduce, avoid and limit impacts on the components of the environment. A Water Management Plan and monitoring program are formulated in this work which describes the obligations of the promoter but with a predefined schedule.

We hope that this book will serve as a basic document for those who want to continue or use the results of this study, even if it is only a modest contribution from us in the study of water management.

\section{Acknowledgements}

The Author would like to acknowledge the support provided by MTH Company, by all the teachers in Department of School of Resources and Safety Engineering in Central South University, especially my supervisor Mr Shi Xiuzhi during the development of the framework and tools, and for providing the financial support for the study.

\section{References}

[1] Panamello, R. and Mohano, O. (1973) Principle and Problems of Groundwater Resource with Cases Example from Developing Countries. Riparian Right and Pollution.

[2] Ezenabor, B.O. (1991) Pollution of Water Whatever Description Is Actionable Riparian Right.

[3] Egboka, B.C.E., Nwankwo, G.I., Orajaka, I.P. and Jiofor, A.O. (1989) Guidelines for Drinking Water Quality (World Water) Illinois Groundwater Pollution. 
[4] Offodile, M.E. (1992) An Approach to Groundwater Study and Development in Nigeria. Mecon Geology and Engineering Services Ltd., Jos.

[5] Oteze, G.E. (1981) Water Resources in Nigeria Environment. Vol. 3, Springer, Bertlin Heidelberg, 177-184.

[6] World Health Organisation (1994) International Standard for Drinking Water. 8th Edition, Geneva, 53 p.

[7] United State Environmental Protection Agency (2012) Edition of the Drinking Water Standards and Health Advisories.

[8] National Agency for food and Drugs Administration and Control, Nigeria (2008) To Safeguard the Public Health of the Nation.

[9] Hem, J.D. (1970) Groundwater Hydrology. McGraw-Hill, Kogakusha, LTD, Tokyo, 480 p.

[10] ANDRIAMAROFAHATRA J.; Boisse H.; Nicollet C., 1990: U-Pb dating on monazites and zircons of the last major granulitic tectono-metamorphic episode in southeastern Madagascar. Accounts of the Paris Academy of Sciences, 310, II. 1643-1648pp.

[11] COLLINS and WINDLEY, 2002: The tectonic evolution of central and northern Madagascar and its place in the final assembly of Gondwana. Journal of Geology. 325 - 340 pp.

[12] COLLINS A. S .; PISAREVSKI S. A., 2005: Amalgamating Eastern Gondwana: The evolution of Circum-Indian Orogens. Earth-Science Reviews 71 (2005). 229-270pp.

[13] COLLINS A. S., 2006: Madagascar and the amalgamation of central Gondwana. Gondwana research 9 (2006). 3 $16 p p$.

[14] COLLINS A.S .; SANTOSH M .; BRAUN; CLARK C., 2007: Age and sedimentary origin of the Southern Granulites, South India: U-Th-Pb SHRIMP secondary ion mass spectrometry. Precambrian Research 155 (1-2). 125-138pp.

[15] COULIBALY L., 1996: Structural interpretation of lineaments by satellite image processing: the case of the Abitibi and opatica subprovinces (Quebec). Final dissertation. 61-65pp.

[16] COX R .; AMSTRONG R .; ALSWAL L., 1998: Sedimantology, geochronology and provenance of the proterozoic Itremo group, Central Madagascar and applications for pre.Gondwana paleogeograph, Journal of the Geological Society. London, vol. 155. 1009 - 1024 pp.

[17] FOUCAULT A.; RAOULT J., 2000: Dictionary of Geology; Masson 5th edition. 352P.

[18] Chikuni, O., A. Polder, J.U., Skaare and Nhachi, C.F.B. 1997. An Evaluation of DDT and DDT Residues in Human Breast Milk in the Kariba Valley of Zimbabwe. Bulletin Environmental Contamination Toxicology, pp. 776-778.

[19] Conboy, M. J. and M. J. Goss. 2000. Natural protection of groundwater against bacteria offecal origin. Journal of Contaminant Hydrology 43:1-24.

[20] Davis, R. and R.Hirji (eds). 2003a. Water Quality Management: Assessment and Protection. Water Resources and Environment Technical Note D.1. World Bank, Washington DC.

[21] Davis, R. and R.Hirji (eds). 2003b. Water Quality Management: Wastewater Treatment. Water Resources and Environment Technical Note D.2. World Bank, Washington DC.

[22] Davis, R. and R. Hirji (eds). 2003c. Water Quality Management: Non-point Source Pollution. Water Resources and Environment Technical Note D.3. World Bank, Washington DC.

[23] Fu, J., Zhou, Q., Liu, W., Wang, T., Zhang, T. and G. Jiang. 2008. High levels of heavy metals in rice (Oryzasativa L.) from a typical E-waste recycling area in southeast China and its potential risk to human health. Chemosphere 71: 1269-1275.

[24] Hutton, M. 1987. Human Health Concerns of Lead, Mercury,Cadmium and Arsenic in Hutchinson T.C. and Meema K. M. (eds) Lead, Mercury, Cadmium and Arsenic in the Environment. John Wiley, London.

[25] Ministry of Environmental Protection. Technical Guidelines for Environmental Impact Assessment - Groundwater Environment. HJ610-2011. 2011.2 Released

[26] Department of Supervision and Management, National Environmental Protection Agency. Training Materials for Environmental Impact Assessment. Chemical Industry Press. 2000:176-190

[27] The Fourth Group of Hydrogeology, Hebei Geological Bureau. Handbook of Hydrogeology. Beijing: Geological Publishing House. 1978: 69-122, 341-385

[28] Editor-in-Chief Liu Zhengfeng. Hydrogeology Handbook. Silver Sound Press. 2005:1013-1040 